\title{
UIVERSITY OF OSLO
}

Department of Informatics

Modeling and Analysis of a $40 \mathrm{GHz}$ MIMO

System for

Fixed Wireless Access

Research Report 353

Frode Bøhagen

Pål Orten

Geir E. Øien

Isbn 82-7368-310-9

Issn $\quad 0806-3036$

March 2007






\title{
Modeling and Analysis of a $40 \mathrm{GHz}$ MIMO System for Fixed Wireless Access
}

\author{
Frode Bøhagen, Pål Orten, and Geir E. Øien
}

\begin{abstract}
The throughput of a possible future fixed wireless access multiple-input multiple-output (MIMO) system operating at high frequencies is investigated. We extend our previous theoretical work on MIMO for line-of-sight (LOS) channels to show that a considerable gain in throughput is achieved compared to single-input single-output transmission for a practical system, which among other things is subject to antenna array size constraints. In our investigation we apply a propagation model applicable for high frequencies, where often LOS is required for sufficient coverage, and where weather phenomena like rain have considerable impact on the quality of the radio link. A state of the art transmission scheme is utilized with eigenmode transmission, low density parity check coded modulation, power control, and bit loading. The performance is evaluated both with respect to throughput and Shannon capacity.
\end{abstract}

\section{INTRODUCTION}

Fixed wireless access (FWA) is a flexible and cost efficient way to bring network applications such as Internet and telephony to organizations and homes. This technology requires no expensive and time consuming digging of cables, and is easily scalable with regard to the applications' demand. Some predictions on the role of FWA in the future communication environment are given in [1], [2], [3].

Most research efforts in the field of multiple-input multiple-output (MIMO) communications utilize the fading given by the multipath environment, while FWA at high frequencies usually requires a strong line-of-sight (LOS) component for sufficient coverage. However, some recent papers have shown the possibility of getting high MIMO gain for LOS channels [4], [5], [6].

This paper contains a more comprehensive presentation of the results we first presented in [7], where we combine the LOS MIMO ideas with propagation models for high frequency FWA. For such systems phenomena such as for instance rain has an important impact on 
the quality of the received signal. The transmission scheme utilized is based on eigenmode transmission with bit loading and power allocation. We present a novel analysis of constraints on maximum array size with regards to throughput for a LOS based MIMO system. The performance is evaluated with respect to Shannon capacity, and with respect to throughput when introducing a quality of service (QoS) requirement and low density parity check (LDPC) coded modulation. Other simulation results based on the optimal design principle for LOS MIMO were recently reported in [8], [9], where a ray-tracing model is applied to investigate the performance of a $5.2 \mathrm{GHz}$ system in an urban transmission environment.

The rest of the paper is organized as follows. Section II presents the system model with the transmission scheme applied. Then in Section III the channel model and signal-to-noise and-interference ratio (SNIR) calculations are described, while results and conclusions are given in Section IV and Section V respectively.

\section{SYSTEM MODEL}

When employing multiple antennas at the transmitter $(\mathrm{Tx})$ and receiver $(\mathrm{Rx})$ in a multiuser system, it has been shown that the optimal with respect to sum capacity is to do beamforming when the number of users becomes large [10]. This transmission technology is called spatial division multiple access (SDMA), and it makes it possible to do communication between several subscriber units (SUs) and the base station (BS) at the same time, and in the same frequency band. However, in this paper we have chosen to investigate the performance of a single MIMO link, where the spatial domain is exploited by employing spatial multiplexing. This communication link could of course be part of a multiuser system, for example by dividing the channel into time slots, i.e. time division multiple access (TDMA), and dividing these time slots between the SUs. Consequently, the performance results presented for the single link can easily be extended to performance results for the whole multiuser system when employing TDMA and spatial multiplexing.

Even though fixed wireless LOS transmission at high-frequencies is generally not exposed to rapid channel variations, it can be argued that it is beneficial to utilize channel state information (CSI) feedback to the $\mathrm{Tx}$ in some scenarios. For example, a rain event can introduce a severe change in the channel characteristics as described in Section III. The CSI needed at the Tx for our system is the MIMO channel matrix characteristics and the subchannels' SNIR. The channel estimation problem will not be studied in this paper, i.e. we will assume that perfect CSI is available at both the Tx and the Rx. 


\section{A. Transmission on eigenmodes}

Transmission over a slowly varying and frequency-flat fading MIMO channel in complex baseband can be expressed as [11]

$$
\mathbf{r}=\sqrt{\chi} \cdot \mathbf{H x}+\mathbf{n},
$$

where $\mathbf{r} \in \mathbb{C}^{M \times 1}$ is the received signal vector, the factor $\chi$ is the common power gain over the channel, $\mathbf{H} \in \mathbb{C}^{M \times N}$ is the normalized channel matrix, $\mathbf{x} \in \mathbb{C}^{N \times 1}$ is the transmitted signal vector, and $\mathbf{n} \in \mathbb{C}^{M \times 1}$ is the complex additive white Gaussian noise (AWGN) vector. The elements in $\mathbf{H}$ links the $N$ Tx antennas with the $M$ Rx antennas (transfer function). It is assumed that all the subchannels experience the same path loss. This common path loss is incorporated in the $\chi$ factor. Furthermore, the channel matrix is normalized, which means that the elements have an average power of one.

The CSI assumed available at both sides of the communication link is utilized to transmit on the eigenmodes. By doing a singular value decomposition (SVD) [12, p.54], $\mathbf{H}$ can be expressed as $\mathbf{H}=\mathbf{U} \boldsymbol{\Sigma} \mathbf{V}^{H}$, where $\mathbf{U} \in \mathbb{C}^{M \times M}$ is a unitary matrix, $\boldsymbol{\Sigma} \in \mathbb{R}^{M \times N}$ is a diagonal matrix with the singular values of $\mathbf{H}$ as its elements (denoted $\sigma_{H, i}$ and descending sorted from upper left to lower right), and $\mathbf{V} \in \mathbb{C}^{N \times N}$ is a unitary matrix containing the eigenvectors of $\mathbf{H}$. Eigenmode transmission is accomplished by pre-multiplying the symbol vector to be sent with the unitary matrix $\mathrm{V}$, i.e. transmitting on the eigenvectors of the channel matrix. The resulting subchannels are orthogonal to each other, which means there are no cross-talk between them.

The CSI will also be employed to introduce power allocation on the eigenmode channels. This can be modeled by multiplying the symbol vector $\mathbf{s}$ with a diagonal matrix $\mathbf{W} \in$ $\mathbb{R}^{N \times N}$. The diagonal elements of the power allocation matrix is denoted $\sigma_{W, i}$, and they are subject to the total Tx power constraint $\sum_{i=1}^{N} \sigma_{W, i}^{2}=P$. By doing this preprocessing of the symbol vector, and utilizing the SVD of $\mathbf{H}$, we get the following expression for the MIMO transmission:

$$
\begin{aligned}
\mathbf{r} & =\sqrt{\chi} \cdot \mathbf{H x}+\mathbf{n} \\
& =\sqrt{\chi} \cdot \underbrace{\mathbf{U} \mathbf{\Sigma} \mathbf{V}^{H}}_{\mathbf{H}} \underbrace{\mathbf{V} \mathbf{W}}_{\mathbf{x}}+\mathbf{n} \\
& =\sqrt{\chi} \cdot \mathbf{U} \mathbf{\Sigma} \mathbf{W} \mathbf{s}+\mathbf{n} .
\end{aligned}
$$

Defining $\tilde{\mathbf{r}}=\mathbf{U}^{H} \mathbf{r}$ and $\tilde{\mathbf{n}}=\mathbf{U}^{H} \mathbf{n}$, we obtain

$$
\tilde{\mathbf{r}}=\sqrt{\chi} \cdot \Sigma \mathbf{W s}+\tilde{\mathbf{n}},
$$



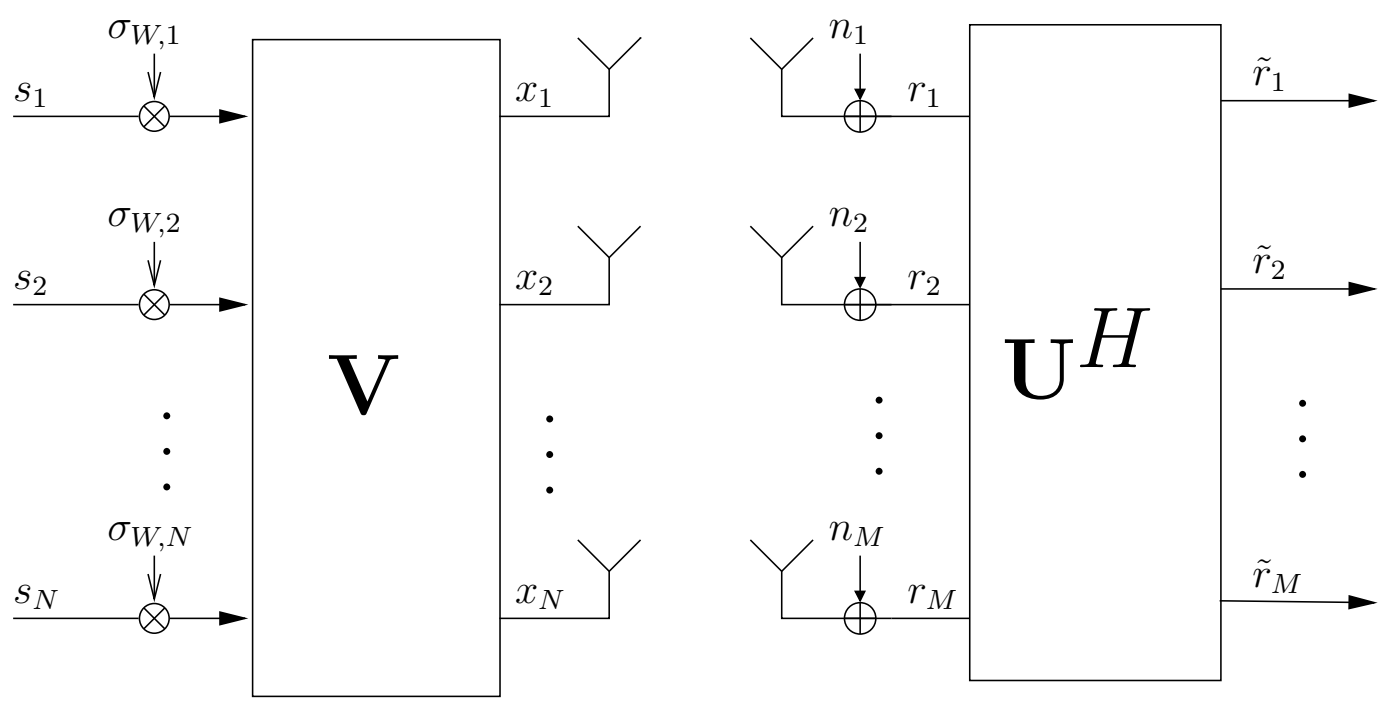

Fig. 1. Eigenmode transmission system with power allocation.

where $\tilde{\mathbf{r}} \in \mathbb{C}^{M \times 1}$ is the vector obtained after combining at the Rx. It is easy to show that $\tilde{\mathbf{n}}$ has the same statistical properties as $\mathbf{n}$ (same distribution).

From (2) we observe that the first $J$ elements of the $\tilde{\mathbf{r}}$ vector can be viewed as parallel single-input single-output (SISO) AWGN channels, each with gain $\sqrt{\chi} \cdot \sigma_{H, i} \cdot \sigma_{W, i}$, where $J=\operatorname{rank}(\mathbf{H})^{1}$. The remaining $M-J$ elements of $\tilde{\mathbf{r}}$ contain no information only noise. Even though $\sigma_{H, i} \neq 0$ for $i \in\{1, \ldots, J\}$, it is not always beneficial to utilize all $J$ equivalent SISO channels when employing the waterfilling (WF) power allocation scheme, as will be described in Section II-B. In Figure 1, the transmission system described is illustrated, while the equivalent form from (2) is shown in Figure 2.

\section{B. Power allocation and bit loading}

As mentioned earlier the system has total available Tx power $P$. This power should be distributed between the subchannels in a smart way. Usually a transmission system is subject to some kind of QoS-constraint given by the application. In this paper we introduce a biterror-rate (BER) constraint, i.e. each subchannel should have a instantaneous BER lower than a given threshold, $\mathrm{BER}_{0}$.

\footnotetext{
${ }^{1}$ The rank of a matrix is equal to the number of independent rows and/or columns of the matrix, and is equivalent to the number of non-zero singular values.
} 

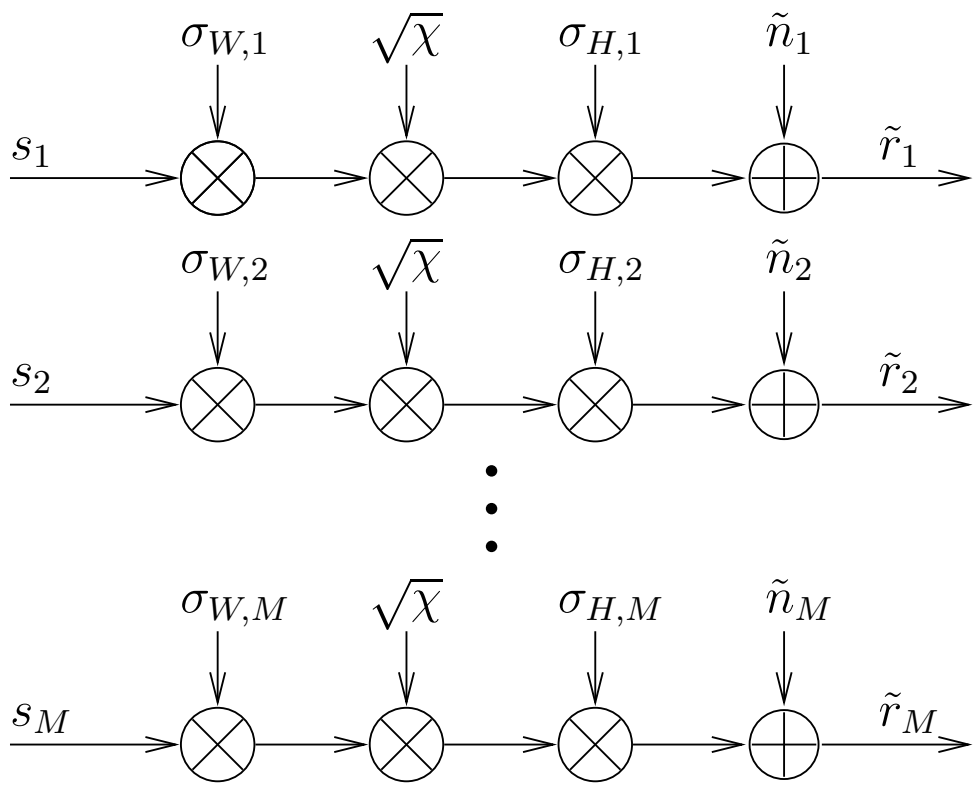

Fig. 2. Equivalent eigenmode transmission model with power allocation.

One simple but not optimal way to use the Tx power is to divide it equally between the subchannels. The bit loading can then be performed by maximizing the bit rate on each subchannel with the BER-constraint based on the subchannels' SNIRs.

A better but more complex scheme was presented in [13]. It is referred to as QoS-based WF. The method starts by dividing the total Tx power between the subchannels according to the WF distribution, and is further constructed with the objective to use minimum power to achieve the BER-threshold on each subchannel, and re-distribute the residual power in a smart way. The step by step procedure is described in [13].

\section{Coding and modulation}

In this paper we utilize LDPC codes with QAM/PSK modulation. LDPC codes are block codes proposed by Gallager in 1963 [14]. Its main features is the high performance together with the iterative decoding scheme, where the complexity only grows linearly with the block length. As an example of LDPC codes we use the results from [15, Table 3], where the block length is 200 QAM/PSK channel symbols. We introduce a QoS-requirement of BER less than $10^{-6}$. The SNIR thresholds for where each transmission mode starts to fulfill the BER-constraint are given in Table I. Furthermore, we assume transmitting at the maximum inter-symbol-interference free symbolrate for a bandpass signal, i.e. $1 \mathrm{symb} / \mathrm{s} / \mathrm{Hz}$. 
TABLE I

SNIR THRESHOLDS FOR LDPC CODED QAM/PSK TRANSMISSION MODES WITH BER B $_{0}=10^{-6}$.

\begin{tabular}{|c|c|c|c|}
\hline Number & Code rate & $\begin{array}{c}\text { Bit rate } \\
\text { (infobit/symbol) }\end{array}$ & $\begin{array}{c}\text { SNIR threshold } \\
\text { in } \mathrm{dB}\end{array}$ \\
\hline 1 & $1 / 2$ & 1 & 3.84 \\
2 & $2 / 3$ & 2 & 9.27 \\
3 & $3 / 4$ & 3 & 12.59 \\
4 & $4 / 5$ & 4 & 15.93 \\
5 & $5 / 6$ & 5 & 18.83 \\
6 & $6 / 7$ & 6 & 21.86 \\
7 & $7 / 8$ & 7 & 24.98 \\
8 & $8 / 9$ & 8 & 27.92 \\
\hline
\end{tabular}

\section{LOS based MIMO transmission}

Usually MIMO is employed to utilize the decorrelation between the subchannels that is introduced by a rich multipath environment. In [4] and [5] it was shown that a pure LOS channel can give a large MIMO gain by placing the antennas in the Tx and Rx in a smart way. In [6] we studied the uniform linear array (ULA) case, and a more general geometrical model was introduced where new parameters were incorporated. The optimal design parameters resulting in an orthogonal LOS matrix were given by

$$
d_{t} d_{r}=\frac{\lambda R}{V \cos \theta_{t} \cos \theta_{r}}
$$

where $d_{t}$ and $d_{r}$ are the antenna separation distance at the Tx and the Rx respectively, $\lambda$ is the wavelength, $R$ is the distance between Tx and $\mathrm{Rx}, V=\max (M, N)$, and $\theta_{t}$ and $\theta_{r}$ are the relative angles between the arrays [6]. From the equation we see that the product between $d_{t}$ and $d_{r}$ is the key design quantity. This product is referred to as the antenna separation product (ASP).

In [6] the sensitivity with regards to deviation from the optimal ASP value in (3) was studied, based on a deviation factor $\eta$. The deviation factor is defined as the ratio between the orthogonal ASP, i.e. RHS of (3), and the actual ASP. For example, a deviation factor of 2 can be interpreted as an actual ASP of half the optimal, i.e. too small antenna arrays. It was shown that the LOS MIMO scheme actually performs better than MIMO based on uncorrelated Rayleigh subchannels for small deviation factors. 


\section{E. Shannon capacity}

Optimal power allocation with respect to capacity when the channel is known at the Tx is in accordance with the WF scheme [11]. The capacity is then given by

$$
C_{W F}=\sum_{i=1}^{J}\left(\log _{2}\left(\xi \frac{\chi \cdot \sigma_{H, i}^{2}}{\sigma_{n}^{2}}\right)\right)_{+} \quad \mathrm{bit} / \mathrm{s} / \mathrm{Hz},
$$

where $\sigma_{n}^{2}$ is the noise (and interference) power at one $\operatorname{Rx}$ antenna, $(x)_{+} \triangleq \max (0, x)$, and $\xi$ is chosen to satisfy

$$
\sigma_{W, i}^{2}=\left(\xi-\frac{\sigma_{n}^{2}}{\chi \cdot \sigma_{H, i}^{2}}\right)_{+} \quad i \in\{1, \ldots, J\},
$$

with the constraint on the total Tx power $\sum_{i=1}^{N} \sigma_{W, i}^{2}=P$, as described earlier $\left(\sigma_{W, i}=0\right.$ for $i \in\{J+1, \ldots, N\}$ since the channel gain is zero for these subchannels).

\section{ChANNEL MODELING}

In Figure 2 there are three parameters that are dependent on the transmission environment, i.e. $\chi, \sigma_{H, i}$ and $\tilde{n}_{i}$. In this section we will discuss them further, and present models that is applicable for our system. To keep the analysis clear and easy-to-follow, we restrict the propagation modeling to the influence of path loss and rain. We acknowledge that there are other channel effects that are important with respect to propagation at high frequencies as well, e.g. scintillation and vegetation [16], but in our opinion the results based on our simplified model still give important insight into the performance of LOS MIMO systems at high frequencies. The section is rounded off by giving an expression for the SNIR of the communication link.

\section{A. Propagation model}

To predict the signal attenuation for our high frequency system we utilize a propagation model called Free space + RMD [17, p.98], where RMD is short for Reflection/Multiple Diffraction. This propagation model is based on the basic principles of physics rather than statistical outcomes from experiments. Only the LOS attenuation part of the model will be used, and it is given by [17, p.102]

$$
L_{\mathrm{LOS}}=32.45+20 \log f+20 \log R+A_{r}+A_{f r} .
$$

Here, $f$ is the frequency in $\mathrm{GHz}, A_{r}$ is a reflection attenuation term, and $A_{f r}$ is the attenuation because of Fresnel zone obstruction. However, for simplicity the two attenuation terms, $A_{r}$ 
and $A_{f r}$, are set to zero $\mathrm{dB}$. This is common for the $A_{r}$ term when the reflection point is not precisely known. To justify the removal of $A_{f r}$, we assume that the signal path has sufficient clearance from potential obstacles. However, if one is to analyse a specific communication link, one should verify that this actually is the case.

If the transmitted signal is exposed to rain, additional attenuation is introduced. To model the rain attenuation we use Crane's rain fade model. The model is given as follows [17, p.144]

$$
\begin{aligned}
& L_{\text {rain }}=k R_{\text {rain }}^{\alpha}\left(\frac{e^{\mu \alpha r}-1}{\mu \alpha}-\frac{b^{\alpha} e^{c \alpha r}}{c \alpha}+\frac{b^{\alpha} e^{c \alpha \tilde{R}}}{c \alpha}\right) d B \\
& L_{\text {rain }}=k R_{\text {rain }}^{\alpha}\left(\frac{e^{\mu \alpha \tilde{R}}-1}{\mu \alpha}\right) d B \quad \text { for } r \leq \tilde{R} \leq 22.5 \mathrm{~km} \\
& \text { for } r>\tilde{R}
\end{aligned}
$$

where

$$
\begin{aligned}
\mu & =\frac{\ln \left(b e^{c r}\right)}{r} \\
b & =2.3 \cdot R_{\text {rain }}^{-0.17} \\
c & =0.026-0.03 \cdot \ln \left(R_{\text {rain }}\right) \\
r & =3.8-0.6 \cdot \ln \left(R_{\text {rain }}\right) \\
R_{\text {rain }} & =\text { rain rate in } \mathrm{mm} / \mathrm{h} \\
\tilde{R} & =\text { path length in } \mathrm{km} .
\end{aligned}
$$

Values for the two regression coefficients, $k$ and $\alpha$, which are functions of frequency and polarization, can be found in [18].

Now, the power gain from the Tx to the $\mathrm{Rx}$ at a distance $R$ is given by

$$
\chi(d B)=G_{t}(d B)+G_{r}(d B)-L_{\mathrm{LOS}}-L_{\mathrm{rain}},
$$

where $G_{t}$ and $G_{r}$ are the Tx and Rx antenna gain respectively.

\section{B. Short term variation}

In addition to the extra attenuation, rain introduces a short term stochastic behavior for the received signal. In [19] it was suggested that the received amplitude follows a Ricean distribution. Ricean MIMO channels can be modeled as a sum of two channel matrices, one LOS and one non-LOS (NLOS), where the Ricean $K$-factor is defined as the ratio between 
the power of the two [4]. As mentioned earlier the channel matrix $\mathbf{H}$ is assumed to be normalized, i.e. the common path loss is moved out of the matrix. Under a rain event the channel matrix can thus be modeled as

$$
\mathbf{H}=\sqrt{\frac{K}{K+1}} \mathbf{H}_{\mathrm{LOS}}+\sqrt{\frac{1}{K+1}} \mathbf{H}_{\mathrm{NLOS}} .
$$

Here, $K$ is the Ricean $K$-factor which is given by [19]

$$
K(d B)=\left\{\begin{array}{lll}
16.88-0.04 R_{\text {rain }} & \mathrm{dB} & R_{\text {rain }} \neq 0 \\
\infty & \mathrm{dB} & R_{\text {rain }}=0
\end{array}\right.
$$

As observed from (9) and (10) the channel matrix becomes a pure LOS matrix, and thus deterministic, when there is no rain.

To be able to investigate the MIMO system the elements of $\mathbf{H}_{\mathrm{LOS}}$ and $\mathbf{H}_{\mathrm{NLOS}}$ have to be determined. For the LOS matrix the geometrical model presented in [6] is applied. This model is based on ULAs at both sides, and the arrays can take any orientation. The amplitudes of the matrix elements are equal to one as described earlier, while a ray-tracing technique is used to find the different phases over the Rx array. The reader is referred to [6] for further details. The NLOS matrix elements are independent and identically distributed complex Gaussian (Rayleigh matrix), with variance equal to one because of the normalization. Consequently, it is assumed that there are no correlation between the different NLOS components.

The equations above show that the performance of a radio link operating at high frequencies is heavily dependent on the rain rate. Rain rates are usually given by the probability that the rain exceeds a given value in millimeters per hour $(\mathrm{mm} / \mathrm{h})$. Areas that have high total rainfall could have fewer deep rain fade periods than areas with lower total rainfall. This is because it is the intensity of the rain that decides the fading depth. Worldwide rain rate contour maps can be found in the literature, e.g. in [20].

\section{Signal-to-noise and-interference ratio}

In Section II-A the channel was modeled as an AWGN channel, i.e. the radio link is exposed to additive noise. We introduce two different noise sources: thermal noise at the Rx and noise due to interference from co-channel users. The equivalent noise power at the $\mathrm{Rx}$ is given by

$$
\sigma_{n}^{2}=k T_{0} B \cdot F+P_{I} \quad \mathrm{~W}
$$

where $k$ is Boltzmann's constant $\left(1.38 \cdot 10^{-23} \mathrm{~J} / \mathrm{K}\right), B$ is the channel bandwidth, $T_{0}$ is room temperature $(293 \mathrm{~K}), F$ is the Rx noise figure, and $P_{I}$ is the co-channel interference power. 
Consequently, we have modeled the co-channel interference as Gaussian, and even though this is not true in general, it can be argued that it makes sense as the number of interferers increase (Central Limit Theorem). The SNIR for subchannel number $i$ in Figure 2 then becomes

$$
\operatorname{SNIR}_{i}(d B)=20 \log \left(\sigma_{W, i}\right)+20 \log \left(\sigma_{H, i}\right)+\chi(d B)-20 \log \left(\sigma_{n}\right) .
$$

In the results section we will utilize the average received SNIR at one Rx antenna when comparing the performance of different systems, which becomes $\bar{\gamma}=\frac{P \cdot \chi}{\sigma_{n}^{2}}$.

\section{RESULTS}

We start by investigating the link performance as a function of the antenna array design for a fixed average SNIR. The quality of the antenna array design is quantized through the deviation parameter $\eta$, as described in Section II-D. The case investigated could for instance be a SU in a fixed position with a given average SNIR, where we want to explore how the array design affect the performance.

In Figure 3 the throughput change as a function of $\eta$ is illustrated for a $3 \times 3 \mathrm{MIMO}$ system with LDPC coded modulation for both power and bit allocation schemes. The Shannon capacity is also plotted as a reference. From the figure we can clearly see the performance gain by doing a correct antenna array design, as the throughput decreases when moving away from optimal $(\eta=0 \mathrm{~dB})$ with increasing deviation factor. The figure also illustrates the gain by using QoS-based WF in contrast to the equal power allocation scheme.

Figure 4 shows the impact of rain rate on the throughput for the same system as in Figure 3. We observe that when there is no rain the channel is deterministic and so is the throughput (discontinuous curve), while for rain rates grater than zero the channel becomes stochastic and the throughput is averaged over all channel realizations (smooth curves). It is interesting to see how the throughput is increased for large values of the deviation factor as the rain rate increases. This is because $\mathbf{H}_{\mathrm{LOS}}$ becomes low rank for large deviation factors [6], but increasing rain rates increases the influence of $\mathbf{H}_{\mathrm{NLOS}}$, which is high rank. It is important to note that we have isolated this effect by using a constant average SNIR. From Crane's rain fade model we know that an increasing rain rate also increases the attenuation, and this effect is not taken into account her.

We now go one step further and include the propagation model; thus the average SNIR is not constant any more but a function of the distance $R$ and the rain rate $R_{\text {rain }}$. In this 


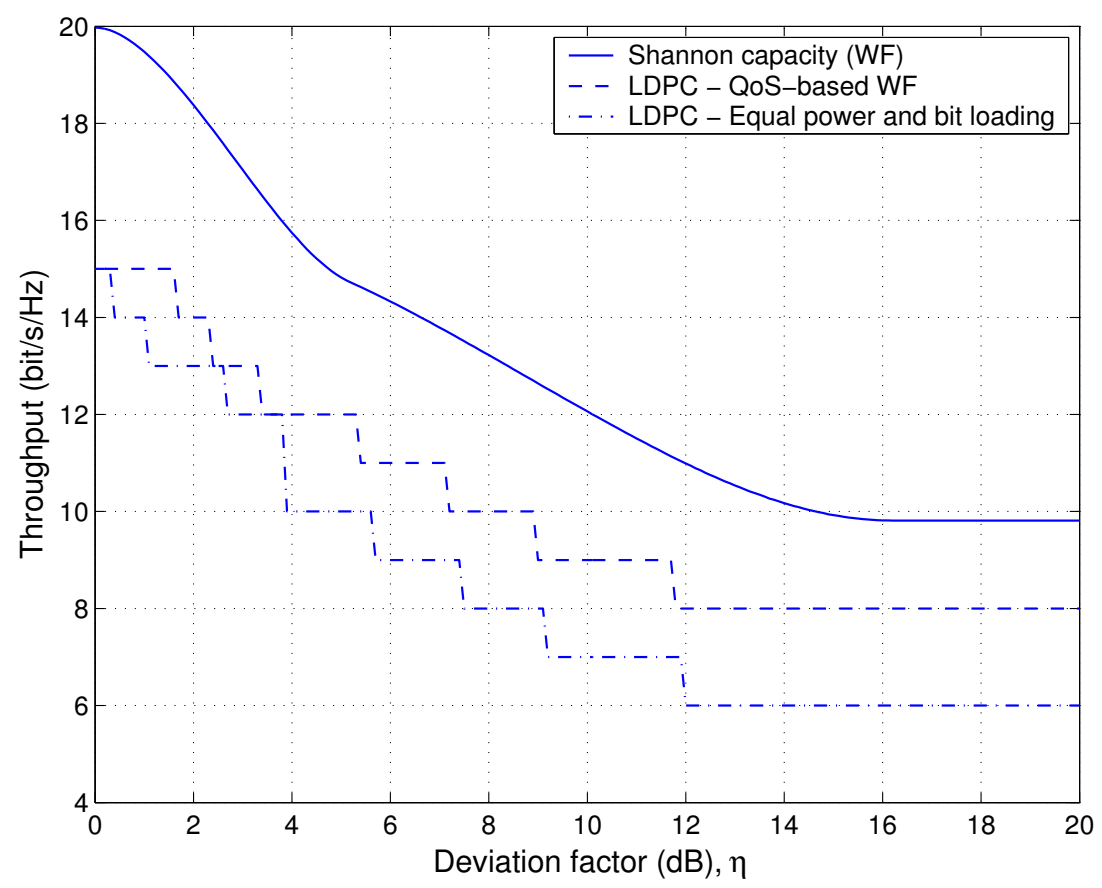

Fig. 3. Throughput as a function of deviation factor $\eta$ for a $3 \times 3$ MIMO system with LDPC coded modulation and different power and bit allocation schemes. No rain situation (pure LOS) and $\bar{\gamma}=20 \mathrm{~dB}$.

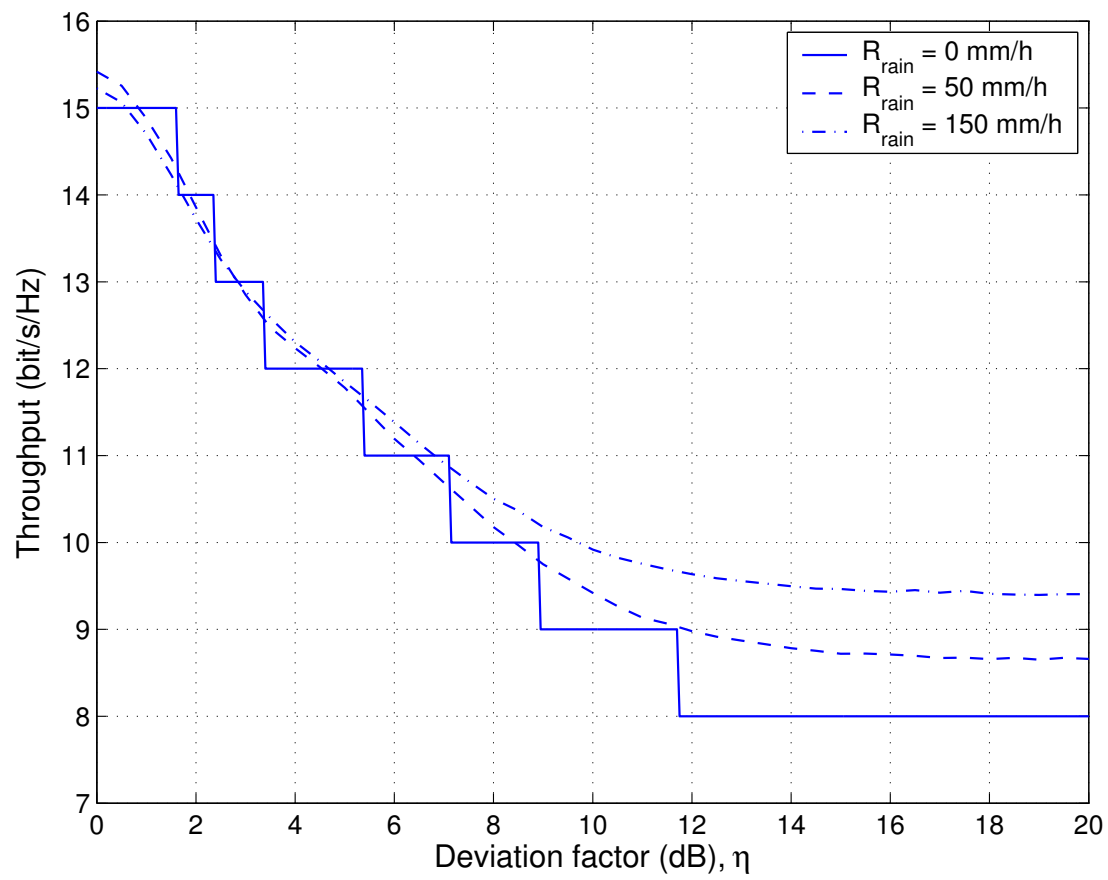

Fig. 4. Throughput as a function of deviation factor $\eta$ for a $3 \times 3$ MIMO system with LDPC coded modulation and QoS-based WF power and bit allocation scheme. Different rain rates and $\bar{\gamma}=20 \mathrm{~dB}$. 


\begin{tabular}{|l|c|}
\hline Parameter & Value \\
\hline Carrier, $f_{c}$ & $40 \mathrm{GHz}$ \\
BS antenna gain, $G_{B S}$ & $15 \mathrm{dBi}$ \\
SU Tx power, $P_{S U}$ & $-6 \mathrm{dBW}$ \\
SU antenna gain, $G_{S U}$ & $32 \mathrm{dBi}$ \\
Receiver Noise figure, $F$ & $8 \mathrm{~dB}$ \\
Bandwidth, $B$ & $28 \mathrm{MHz}$ \\
Number of interfering SUs, $N_{I}$ & 3 \\
Distance to interfering SUs, $R_{I}$ & $5 \mathrm{~km}$ \\
Relative array angle, $\theta_{t}$ and $\theta_{r}$ from $(3)$ & $0^{\circ}$ \\
Regression coefficient, $\alpha_{v}$ & 0.929 \\
Regression coefficient, $k_{v}$ & 0.31 \\
\hline
\end{tabular}

paper we look at the link from the SU to the BS, i.e. uplink. The only difference when investigating the downlink is that we need to change some of the parameter values used; the models remain the same. A set of realistic parameter values are chosen to produce the results. Most of the values are found in [21, App.B], and reproduced for convenience in Table II together with the other parameters employed. The noise and interference power employed to produce the results corresponds to a Rx noise figure of $8 \mathrm{~dB}$, and three identical interfering SUs transmitting with the same power and propagation model (no short term variation) as the desired SU at a distance of $5 \mathrm{~km}$ (because of frequency reuse). For a real system, the number of interferers and distance to the interferers, are dependent on the cellular layout and channel reuse strategy chosen for the system.

In Figure 5 we investigate the effect of constraining the maximum array size. The Shannon capacity is plotted for different array size constraints. The BS array size is fixed while the SU array size is adapted perfectly with distance (Eq. (3)) until it reaches its limit, $\mathrm{SU}_{\max }$, where the ASP is frozen for longer distances (i.e. not optimal design for these distances). Two different MIMO dimensions are plotted together with a SISO system as reference. A first observation is that the figure supports the promising results of LOS based MIMO systems found in [6], and a substantial gain over SISO is predicted. Further, the figure shows that to follow the perfect ASP one needs a larger array for larger MIMO dimensions. For the $5 \times 5$ case the performance for the different array size constraint situations deviate from the 


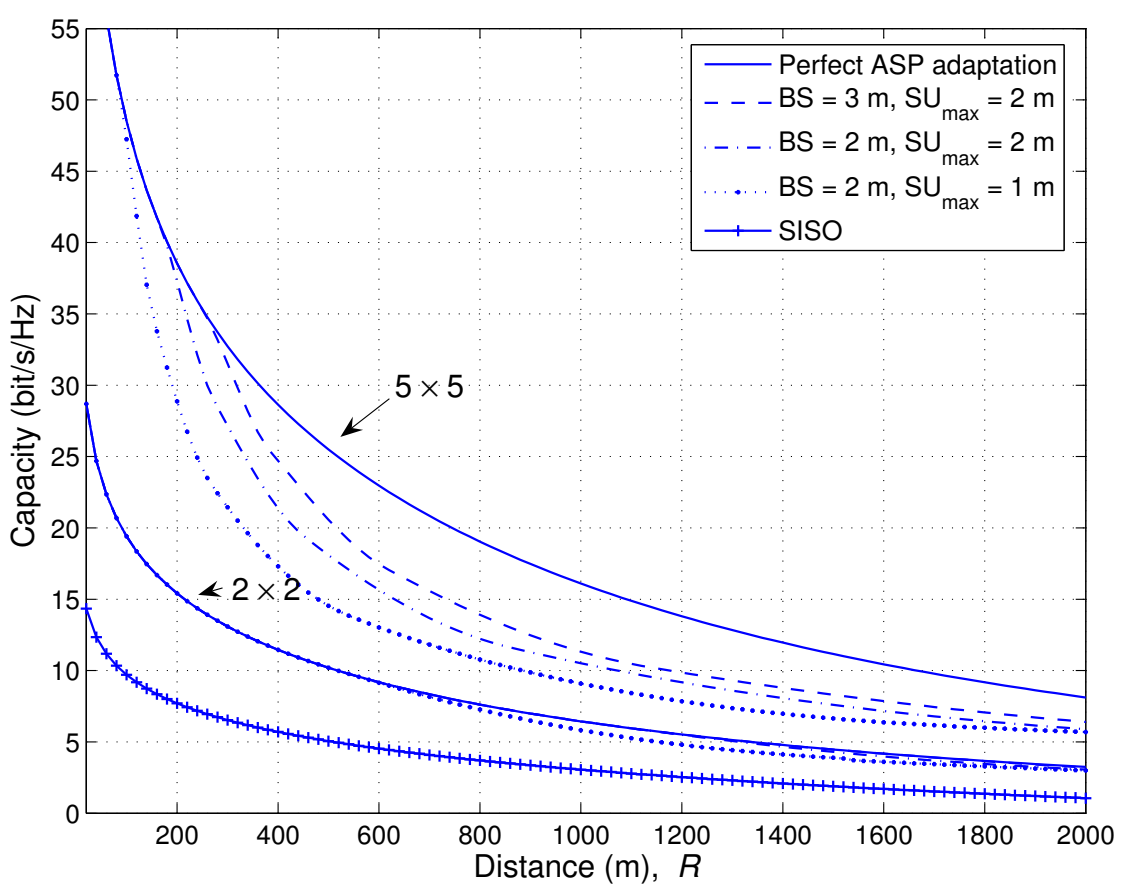

Fig. 5. Shannon capacity as a function of distance for a no rain situation (pure LOS) with array size constraint.

unconstrained performance as the distance increases. The first scenario $\left(\mathrm{BS}=2 \mathrm{~m}, \mathrm{SU}_{\max }=\right.$ $1 \mathrm{~m}$ ) starts to deviate at approximately $100 \mathrm{~m}$, while the deviation begins at $300 \mathrm{~m}$ for the last scenario $\left(\mathrm{BS}=3 \mathrm{~m}, \mathrm{SU}_{\max }=2 \mathrm{~m}\right.$ ). For the $2 \times 2$ case the constrained situations follow the unconstrained much better. The last scenario seems to follow the unconstrained capacity all the way to $2000 \mathrm{~m}$.

In Figure 6 the throughput for a MIMO system employing LDPC coded modulation with QoS-based WF is plotted for different rain rates. A $3 \times 3$ system is considered, and perfect ASP adaptation is assumed. The figure shows that as the rain rate increases the throughput also increases. This can be attributed to the reduced co-channel interference power because of the extra attenuation introduced by the rain, giving an increased SNIR. However, as the rain rate continues to increase we see that the throughput decreases, and for large distances becomes less than that of a rain rate of zero. The reason for this is the large extra attenuation that the rain forces on the desired signal, and even if the interference power is reduced, we still have a constant thermal noise. The transmission scheme used seems to fit best to the propagation scenario in the no rain case, as the throughput adapts best with distance. For the other cases, the throughput is constant equal to $24 \mathrm{bit} / \mathrm{s} / \mathrm{Hz}$ in large regions near the $\mathrm{BS}$, which is maximum throughput for the transmission modes implemented $(3 \cdot 8=24)$. 


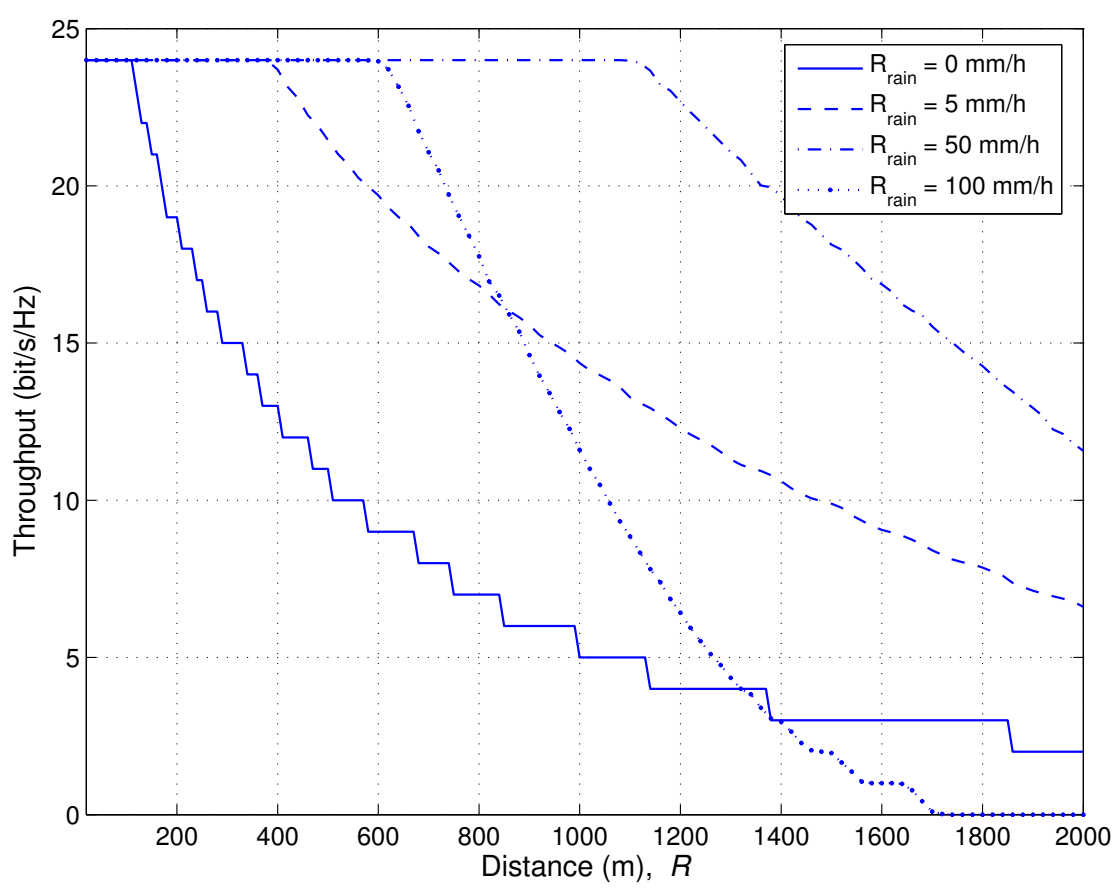

Fig. 6. Throughput as a function of distance for a $3 \times 3$ MIMO system with LDPC coded modulation and QoS-based WF power and bit allocation for different rain rates.

Transmission modes offering higher bit rates could be used here to increase the performance.

\section{CONCLUSION}

The combination of transmission on eigenmodes with power and bit allocation for a LOS based MIMO system is shown to be an interesting way of implementing FWA links at high frequencies. It is shown that much higher throughput is obtained compared to traditional SISO transmission. This is true even if we have some throughput loss when we restrict the array sizes used.

The influence of rain on the radio link is dependent on the combination of several factors. It is beneficial with regards to reduced interference from co-channel users and increased rank for the channel matrix for large deviation factors, but it is negative with respect to extra attenuation of the desired signal.

\section{REFERENCES}

[1] W. Webb, "Broadband fixed wireless access as a key component of the future integrated communications environment," IEEE Commun. Mag., vol. 39, pp. 115-121, Sep. 2001.

[2] T. Tjelta et al., "Future broadband radio access systems for integrated service with flexible resource management," IEEE Commun. Mag., vol. 39, pp. 56-63, Aug. 2001. 
[3] I. Tardy and O. Grøndalen, "On the role of future high-frequency BFWA systems in broadband communications networks," vol. 43, pp. 138-144, Feb. 2005.

[4] P. F. Driessen and G. Foschini, "On the capacity formula for multiple input-multiple output wireless channels: A geometric interpretation,” IEEE Trans. Commun., vol. 47, pp. 173-176, Feb. 1999.

[5] D. Gesbert, H. Bölcskei, D. A. Gore, and A. J. Paulraj, "Outdoor MIMO wireless channels: Models and performance prediction," IEEE Trans. Commun., vol. 50, pp. 1926-1934, Dec. 2002.

[6] F. Bøhagen, P. Orten, and G. E. Øien, "Design of capacity-optimal high-rank line-of-sight MIMO channels," IEEE Trans. Wireless Commun., vol. 6, March 2007 Available from: http://www.unik.no/personer/frodbo/.

[7] F. Bøhagen, P. Orten, and G. E. Øien, "Modeling and analysis of $40 \mathrm{GHz}$ MIMO system for fixed wireless access," in Proc. IEEE VTC, (Stockholm, Sweden), May 2005.

[8] I. Sarris and A. R. Nix, "A line-of-sight optimised MIMO architecture for outdoor environments," (Montreal, Canada), Sept. 2006.

[9] I. Sarris and A. R. Nix, "Performance investigation of a line-of-sight optimised $2 \times 2$ MIMO system," in Proc. International Symp. on Wireless Communication Systems (ISWCS), (Valencia, Spain), Sept. 2006.

[10] W. Rhee and J. M. Cioffi, "On the asymptotic optimality of beam-forming in multi-antenna Gaussian multiple access channels," in Proc. IEEE Globecom, (San Antonio, USA), pp. 891-895, Nov. 2001.

[11] E. Telatar, "Capacity of multiantenna Gaussian channels," AT\&T Bell Laboratories, Tech. Memo, June 1995.

[12] C. W. Therrien, Discrete Random Signals and Statistical Signal Processing. Prentice Hall Signal Processing Series, 1992.

[13] X. Zhang and B. Ottersten, "Power allocation and bit loading for spatial multiplexing in MIMO systems," in Proc. ICASSP, (Hong Kong), pp. 53-56, April 2003.

[14] R. G. Gallager, Low-Density Parity-Check Codes. M.I.T. Press, 1963.

[15] O. Jetlund, G. E. Øien, K. J. Hole, V. Markhus, and B. Myhre, "Rate-adaptive coding and modulation with LDPC component codes," COST 273 TD(02) 108, Sep. 2002.

[16] M. Cheffena, L. E. Bråten, T. Tjelta, and T. Ekman, "Time dynamic channel model for broadband fixed wireless access systems," in Proc. IST Mobile and Wireless Summit, (Myconos, Greece), June 2006.

[17] H. R. Anderson, Fixed broadband wireless system design. Wiley, first ed., April 2003.

[18] ITU-R Rec. P.838-2, Specific attenuation model for rain for use in prediction methods. April 2004.

[19] H. Xu, T. S. Rappaport, R. J. Boyle, and J. H. Schaffner, "Measurements and models for 38-GHz point-to-multipoint radiowave propagation,” IEEE J. Select. Areas Commun., vol. 18, pp. 310-321, April 2000.

[20] ITU-R Rec. P.837-4, Characteristics of precipitation for propagation modeling. April 2003.

[21] IEEE computer society, 802.16.2, IEEE recommended practice for local and metropolitan area networks, coexistence of fixed broadband wireless access systems. March 2004,

Available from: http://standards.ieee.org/getieee802/802.16.html. 